\title{
The Amphiphilic Self-Assembling Peptide EAK16-I as a Potential Hydrophobic Drug Carrier
}

\author{
Jing Wang, ${ }^{1,2}$ Fushan Tang, ${ }^{1,2}$ Feng Li, ${ }^{1,2}$ Juan Lin, ${ }^{1,2}$ Yinghui Zhang, ${ }^{1,2}$ Linfang Du, ${ }^{3}$ and Xiaojun Zhao ${ }^{1,2,4}$ \\ ${ }^{1}$ Institute for Nanobiomedical Technology and Membrane Biology, West China Hospital, Sichuan University, No.1 Ke Yuan 4th Street, \\ Gao Peng Road, Chengdu, Sichuan 610041, China \\ ${ }^{2}$ State Key Lab of Biotherapy of Human Diseases, Cancer Center, West China Medical School, West China Hospital, Sichuan University, \\ No. 37 Guo Xue Xiang, Chengdu, Sichuan 610041, China \\ ${ }^{3}$ College of Life Science, Sichuan University, No. 24, South Section, First Ring Road, Chengdu, Sichuan 610064, China \\ ${ }^{4}$ Center for Biomedical Engineering NE47-378, Massachusetts Institute of Technology, 500 Technology Square, Main Street, Cambridge, \\ MA 02139-4307, USA
}

Correspondence should be addressed to Xiaojun Zhao, xiaojunz@mit.edu

Received 30 March 2008; Accepted 18 June 2008

Recommended by Zhenzhong Yang

It is crucial for hydrophobic drugs to be dissolved and stabilized by carriers in aqueous systems and then to be delivered into target cells. An amphiphilic self-assembling peptide EAK16-I (Ac-AEAKAEAKAEAKAEAK-NH2) is reported here to be able to stabilize a model hydrophobic compound, pyrene, in aqueous solution, resulting in the formation of colloidal suspensions. Egg phosphatidylcholine (EPC) vesicles are used as plasma membranes mimic. Fluorescence data shows that the pyrene is presented in the crystalline form when stabilized by EAK16-I and molecularly migrates from its peptide encapsulations into the membrane bilayers of EPC vesicles when the suspension is mixed with EPC vesicles. Furthermore, the release rate can be controlled by changing peptide-to-pyrene ratio, and the higher ratios lead to the slower release rates due to a thicker encapsulation on the pyrene microcrystals. This demonstrates that EAK16-I, as a promising nanobiomaterial, has the potential to be a hydrophobic compounds carrier.

Copyright (c) 2008 Jing Wang et al. This is an open access article distributed under the Creative Commons Attribution License, which permits unrestricted use, distribution, and reproduction in any medium, provided the original work is properly cited.

\section{INTRODUCTION}

Low water solubility limits the clinical practice of many potent hydrophobic drugs [1]. Therefore, it is crucial in pharmaceutical industry for hydrophobic compounds to be dissolved or stabilized by carriers in aqueous systems and to deliver them into target cells. With good desirable biocompatibility, self-assembling peptides are emerging as promising nanobiomaterials for hydrophobic drug delivery.

The serendipitous discovery of self-assembling peptides has changed our view of peptides as emerging nanobiomaterials [2]. By (re-)designing the amino acid sequences, self-assembling peptides (8-16 residues, $2.5-5 \mathrm{~nm}$ in length) [3], with good biocompatibility, have already been used in versatile biomedical applications, such as cell cultures, tissue engineering, and surface engineering [4-6], while only a few studies had been reported to investigate the applicability of self-assembling peptides as drug carriers [7-9]. To explore this field further, it seems that more studies should be carried out to investigate the potentials of self-assembling peptides as drug carriers. EAK16-I is a peptide made of 16 amino acids with alternating positive and negative charges separated by a hydrophobic amino acid residue (Ala) $[2,10,11]$. At neutral pH, Glu and Lys are negatively and positively charged, respectively. They are believed to form complementary ion pairs when EAK16-I self-assembles into its $\beta$-sheet microstructure which exhibits a hydrophobic and a hydrophilic surfaces. It is expected that the hydrophobic region of EAK16-I can interact with hydrophobic compounds while the charged residues stabilize the complex in aqueous solution.

The purpose of this study is to examine whether EAK16I can stabilize microcrystals of pyrene, a hydrophobic compound, in aqueous solution and deliver them into the membrane of a cell mimic made of the egg phosphatidylcholine (EPC) vesicles. The rate of pyrene migrates from the colloidal crystals stabilized by EAK16-I into membrane bilayer was also studied. We expect to reveal the potential 
of EAK16-I as carrier of hydrophobic compounds and to give some clues to exploit this peptide as hydrophobic drug carrier.

\section{MATERIALS AND METHODS}

\subsection{Materials}

The peptide, EAK16-I ( $1657 \mathrm{~g} / \mathrm{mol}, \mathrm{N}$-terminal acetylation and C-terminal amidation), was synthesized by Shanghai Sangon Biological Engineering Technology \& Services Co., Ltd., Shanghai, China. Egg Phosphatidylcholine (EPC) was purchased from Sinopharm Chemical Reagents Co., Ltd., Shenyang, China. Pyrene (99\%) was obtained from SigmaAldrich, Shanghai, China, and was recrystallized twice from ethanol before experiment. The rest chemicals used in the experiment were acquired from Chengdu Kelong Chemical Reagents Co., Chengdu, China. Deionized water (Elix Water Purification System, Millipore, Mass, USA) was used to prepare all aqueous solutions.

\subsection{Preparation of colloidal suspensions of pyrene crystals}

Weighed amounts of pyrene were added into freshly prepared EAK16-I solutions in a $10 \mathrm{~mL}$ vial. Two peptidepyrene solutions (referred to as EAKI-PY) with different peptide-to-pyrene ratio were prepared to obtain the concentrations of $0.5 \mathrm{mg} / \mathrm{mL}\left(3.02 \times 10^{-4} \mathrm{M}\right)$ for the peptide and $1.00 \mathrm{mg} / \mathrm{mL}\left(4.94 \times 10^{-3} \mathrm{M}\right)$ and $0.267 \mathrm{mg} / \mathrm{mL}$ $\left(1.32 \times 10^{-3} \mathrm{M}\right)$ for pyrene to obtain E05P10 and E05P02 solutions, respectively. Then, these two samples were kept on stirring until equilibrium was reached in about 120 hours. These solutions were deemed at equilibrium when their fluorescence spectrums did not change in 24 hours.

\subsection{EPC vesicle preparation}

EPC $(0.8 \mathrm{~g})$ dissolved in chloroform was added to a $1 \mathrm{~L}$ round-bottom flask. The organic solvent was evaporated by rotary evaporator at room temperature to produce an EPC thin film inside the round-bottom flask [12]. Then, the EPC film was exposed to high vacuum for at least 2 hours to remove residual traces of chloroform at room temperature and was resuspended in $300 \mathrm{~mL}$ of buffer ( $\mathrm{pH} 7.4$, containing $25 \mathrm{mM}$ Tris- $\mathrm{HCl}$ acid and $0.2 \mathrm{mM}$ EDTA) solution. After being bubbled nitrogen, the mixture was sonicated for 30 minutes at $0^{\circ} \mathrm{C}$ in a bath type sonicator at $200 \mathrm{~W}$ output. This was followed by centrifugation at $12000 \times \mathrm{g}$ for 1.5 hours. The supernatant was filtered by $0.44 \mu \mathrm{m}$ and $0.22 \mu \mathrm{m}$ membrane filters in turn. Liposome size was measured by Malvern Zetasizer Nano ZS analyzer. The lipid concentration of EPC vesicles was determined by comparing the difference between mass of the solute of buffer and that of vesicle solution.

\subsection{Steady-state fluorescence measurements}

Fluorescence spectra were recorded on Hitachi F-4500 spectrofluorophotometer at room temperature. Solution samples were operated in a quartz fluorescence cuvette of $1 \mathrm{~cm}^{2}$ cross-section, while solid samples were carried out by using a solid accessory. The following parameters were used in experiments except special indication. Excitation and emission slits were set to $10 \mathrm{~nm}$ and $2.5 \mathrm{~nm}$, respectively. By setting excitation wavelength at $336 \mathrm{~nm}$, the emission fluorescence spectra were scanned from $350 \mathrm{~nm}$ to $650 \mathrm{~nm}$, with scan speed of $240 \mathrm{~nm} / \mathrm{min}$. Excitation spectra were recorded at the selected emission wavelengths $(373 \mathrm{~nm}$ and $470 \mathrm{~nm})$.

Solutions of different pyrene concentration (ranging from $2 \times 10^{-6} \mathrm{M}$ to $1.2 \times 10^{-4} \mathrm{M}$ ) in liposome were prepared for the calibration curve, and the solutions were referred to as EPC-PY solutions. The $I_{m}$ (fluorescence intensity of the pyrene monomer) for the EPC-PY samples was obtained by averaging the mission intensities of the pyrene monomer for the EPC-PY solutions monitored at $373 \mathrm{~nm}$ for 120 seconds. To avoid the influence of xenon lamp fluctuations, the intensity of pyrene monomer standard $\left(I_{s}\right)$ is needed. A degassed and sealed solution of pyrene in ethanol $\left([\mathrm{PY}]=3.96 \times 10^{-5} \mathrm{M}\right)$ was monitored at $373 \mathrm{~nm}$ over 120 seconds, and the measured intensity was then averaged to yield $I_{s}$ after each spectrum of the EPC-PY solutions. The corrected value of the monomer intensity $\left(I_{m} / I_{s}\right)$ for the EPC-PY solutions was gotten by dividing $I_{m}$ by $I_{s}$.

For the experiments of the migration of pyrene from the EAKI-PY solutions into the liposome, the appropriate volume solutions of the EAKI-PY and EPC vesicles were mixed in quartz fluorescence cuvette. The mixtures (referred as EPC-EAKI-PY) were constantly stirred with a small magnetic stirrer during fluorescence measurement. All samples were prepared less than 20 seconds before the fluorescence measurements initiated. To get the kinetic data for pyrene transfer, a time-dependent fluorescence measurement was required by recording $I_{m}$ at $373 \mathrm{~nm}$ for 4 hours at 0.2 -second intervals. Each $I_{m}$-versus-time profile was corrected by dividing $I_{m}$ by $I_{s}$ to avoid lamp fluctuation. Also liposome sizes of solutions of EPC-PY and EPC-EAKIPY were measured by Malvern Zetasizer Nano ZS analyzer.

\subsection{Scanning electronic microscopy (SEM)}

SEM (JSM-5900LV, JEOL Ltd., Tokyo, Japan) was used in the study of the microstructure and dimension of the peptidepyrene complexes. An aliquot of $20 \mu \mathrm{L}$ EAKI-PY solution was placed on a freshly cleaved mica surface. After 10 minutes, the mica surface was rinsed twice with pure water (each time $200 \mu \mathrm{L}$ ) and air-dried overnight. After gilded, the complexes were imaged using the secondary electron (SE2) mode at $20 \mathrm{kV}$.

\subsection{X-ray photoelectron spectroscopy (XPS)}

An aliquot of EAKI-PY solution was placed to cover a freshly cleaved mica surface $(15 \times 10 \mathrm{~mm})$. After air-dried overnight, the XPS spectra of the samples were measured with a XSAM800 (Kratos Ltd., Manchester, UK) by using Al $\mathrm{K} \alpha(1486.6 \mathrm{eV})$ radiation at $12 \mathrm{kV}$ acceleration voltage and an 


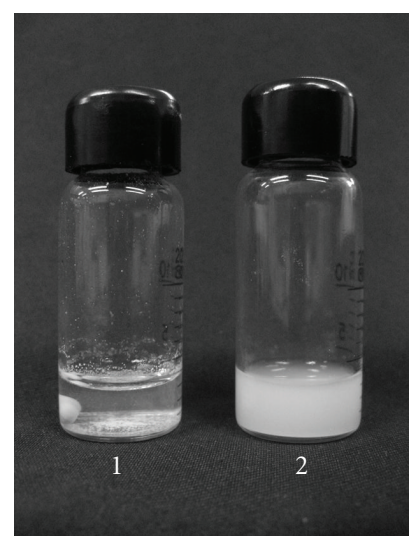

Figure 1: Pyrene $\left([\mathrm{PY}]=4.94 \times 10^{-3} \mathrm{M}\right)$ in pure water $(1)$ and EAK16-I ([EAK16-I $\left.]=3.02 \times 10^{-4} \mathrm{M}=0.5 \mathrm{mg} / \mathrm{mL}\right)$ aqueous solution (2) after stirring for 3 hours.

emission current of $15 \mathrm{~mA}$, and the analyzer mode was fixed analyzer transmission (FAT).

\section{RESULTS AND DISCUSSION}

Pyrene has very low solubility in water (about $6.0 \times 10^{-7} \mathrm{M}$ in the saturated aqueous solution [13]). In the presence of the peptide EAK16-I, a milk-white colloidal suspension was obtained after stirring the EAKI-PY aqueous mixture for 3 hours, while the control sample without peptide still remained transparent with pyrene crystals floating on the top or precipitating at the bottom (see Figure 1). The formation of colloidal suspension can be taken as an initial proof that large amounts of pyrene $\left(4.94 \times 10^{-3} \mathrm{M}\right)$ can be stabilized in water.

In a delivery system, hydrophobic cargos need not only to be stabilized by vehicle in water, but also to be delivered into targets. A hydrophobic compound must be delivered into plasma membrane of the living cell. The essential components of plasma membranes is EPC, hence we utilize EPC vesicles as cell mimic. The lipid concentration of the EPC vesicles used in this study was $7.32 \times 10^{-4} \mathrm{M}$. The average diameters of EPC vesicles in EPC-PY $\left([\mathrm{PY}]=6.52 \times 10^{-5} \mathrm{M}\right)$ and EPC-EAKI-PY $\left([\mathrm{PY}]=5.92 \times 10^{-5} \mathrm{M}, \quad[\mathrm{EAK} 16-\mathrm{I}]=3.52 \times 10^{-6} \mathrm{M}\right)$ solutions were $129 \mathrm{~nm}$ and $131 \mathrm{~nm}$, respectively, which were found to be virtually identical to those of the EPC vesicles $(128 \mathrm{~nm})$.

Figure 2 displays the steady-state fluorescence spectra of the pyrene solutions at equilibrium. The steady-state fluorescence spectra show that the pyrene crystals and the E05P10 solution both exhibit a large amount of pyrene excimer without visible monomer emission (see Figures 2(a) and 2(b)), while pyrene in liposome and the E05P10 solution mixed with the liposome are similar, with large amounts of pyrene monomer and excimer (see Figures 2(c) and $2(\mathrm{~d})) . I_{1} / I_{3}$, the ratio of the intensities of the first peak $\left(I_{1}, 374 \mathrm{~nm}\right)$ and the third peak $\left(I_{3}, 385 \mathrm{~nm}\right)$ of the pyrene monomer, was widely used as a polarity scale to determine the polarity of the pyrene microenvironment [14]. Pyrene in polar solvent like water has an $I_{1} / I_{3}$ ratio about 2.02 and has lower values in apolar environment. The ratios of $I_{1} / I_{3}$ for pyrene in liposome and the E05P10 solution mixed with the liposome are both 1.05. The value variety indicates that pyrene has transferred from the peptide encapsulation into the hydrophobic lipid bilayer. Similar data were acquired with E05P02 solution (data not shown).

The pyrene excimer has two forms: dynamic excimer formed via diffusional encounters between the excited pyrene and a ground-state pyrenean static excimer formed from the direct excitation of ground-state pyrene dimmers in crystalline form [15]. To determine whether the pyrene excimer is dynamic excimer or static excimer, additional experiments, measurement of excitation spectra, must be performed. The static excimer has a red-shift excitation spectrum, and the dynamic excimer has the same excitation spectrum as that of monomer emission [16]. Compared with excitation spectrum monitored at monomer emission, the excitation spectra of pyrene crystals and E05P10 solution monitored at excimer emission red shifts about $45 \mathrm{~nm}$ from about $335 \mathrm{~nm}$ to $380 \mathrm{~nm}$ (see Figures 3(a) and 3(b)). This indicates that the excimers in pyrene crystals and E05P10 solution are static excimers resulting from pyrene molecules preassociated in solid pyrene crystals and that pyrene molecules in pyrene crystals and E05P10 solution were both in crystalline form. Therefore, a further proof that pyrene was stabilized in water via EAK16-I peptide adsorbing onto the surface of pyrene microcrystals was acquired. However, for pyrene in EPC vesicles and EAKI-PY solution mixed with EPC vesicles, no spectra shifts were observed; this suggested that the excimers were formed via diffusional encounter only (see Figures 3(c) and 3(d)) [17, 18]. It also suggested that pyrene was molecularly dissolved inside the EPC vesicle membrane while very few pyrene crystals were expected to remain after the EAKI-PY solution mixing with the EPC vesicles completely. Similar observations were acquired with E05P02 solution (data not shown).

To monitor the migration of pyrene from EAK16-I encapsulation into the EPC vesicles, steady-state fluorescence spectra of PY in EPC-EAKI-PY solution were acquired at various time intervals over a total period of 4.5 hours after mixing EAKI-PY with liposome. All the $I_{1} / I_{3}$ values of these spectra were 1.05; this demonstrated that the pyrene monomers were always located inside the hydrophobic lipid bilayer [14]. As shown in Figure 2, the fluorescence signal of the pyrene excimer can arise from those formed inside the pyrene crystals and the EPC vesicle membrane, whereas that of the pyrene monomer had only one source. Consequently, to monitor the transfer of pyrene during experiment more precisely, the monomer intensity was recorded to get $I_{m}$ versus-time profile over a period of 4 hours with 0.2 seconds intervals at an emission wavelength of $373 \mathrm{~nm}$. Two profiles were shown in Figure 4. Profile 1 represents the release of pyrene from E05P10 solution into EPC vesicles with the final pyrene concentrations of $1.3 \times 10^{-5} \mathrm{M}$. Profile 1 obtained at low pyrene concentration exhibits a continuous increase of $I_{M}$ with time during the first half hour and reaches a plateau after close to 1 hour. Profile 2 represents the release of 


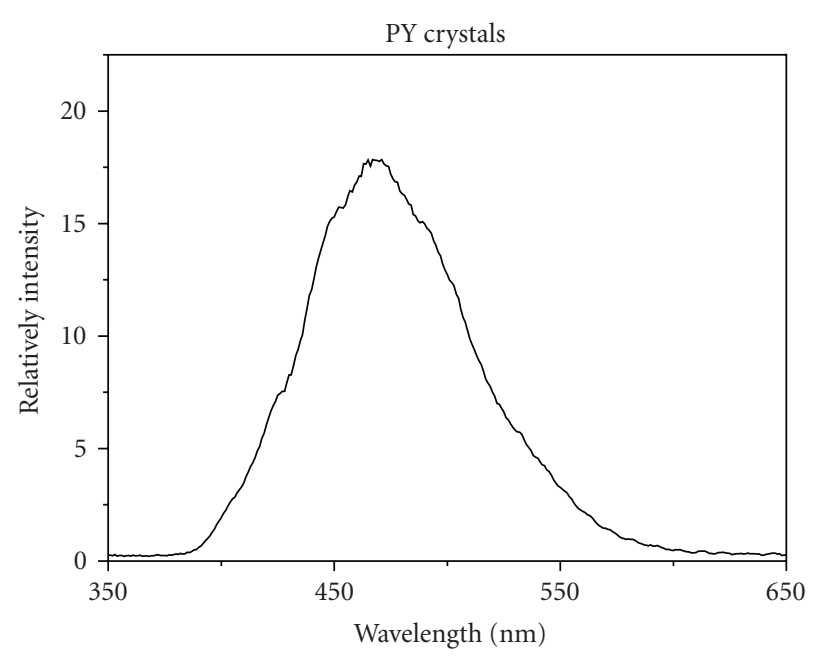

(a)

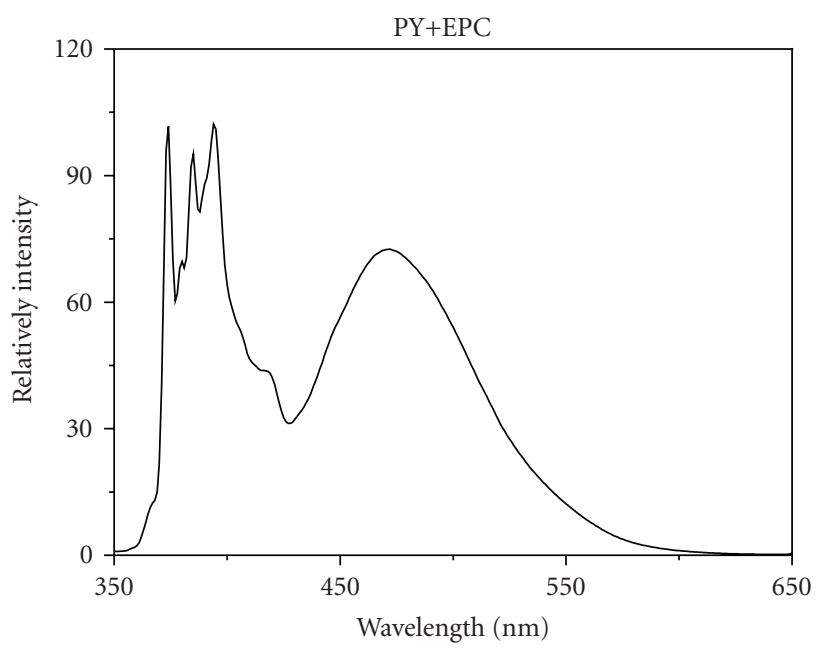

(c)

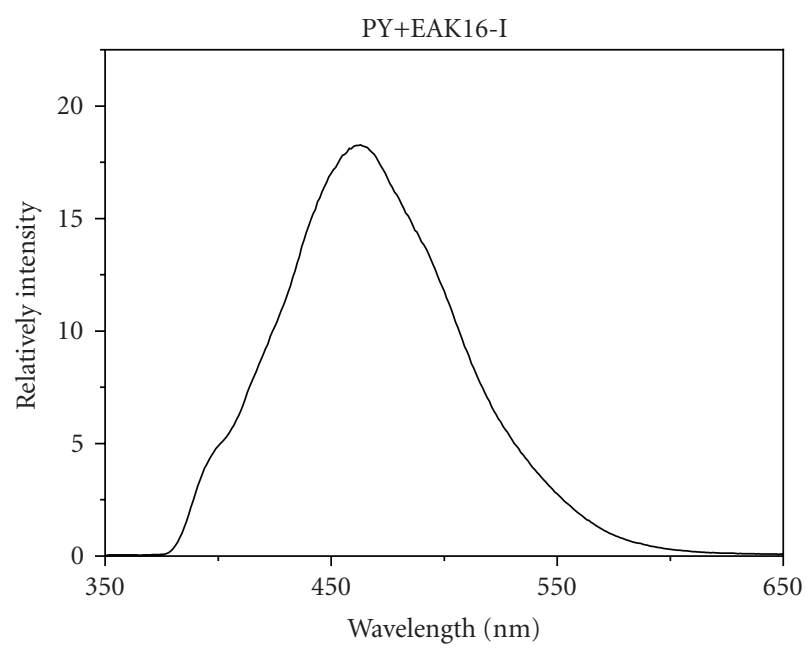

(b)

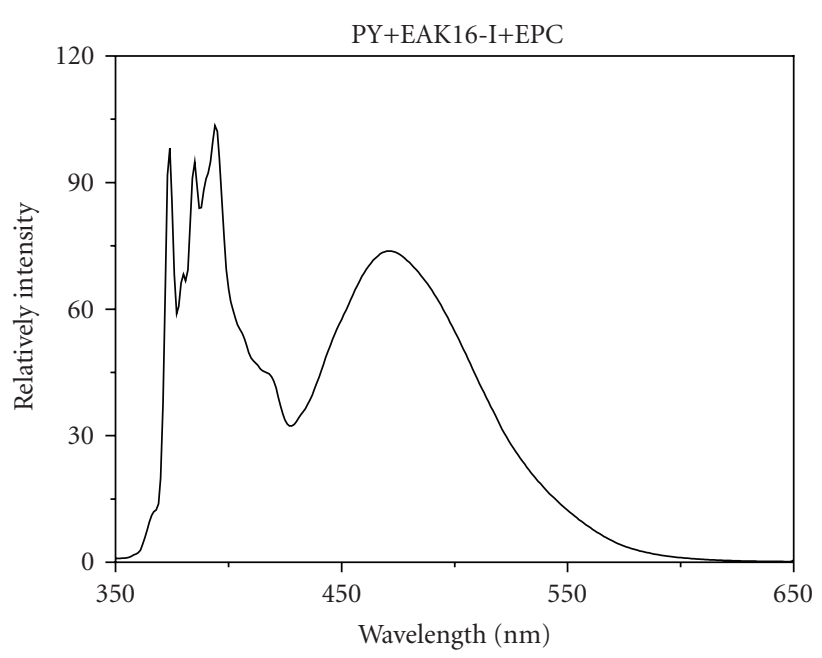

(d)

FIGURE 2: Steady-state fluorescence emission spectra of (a) solid pyrene crystals, (b) E05P10 solution ([PY] $=4.94 \times 10^{-3} \mathrm{M}$, $\left[\right.$ EAK16-I] $\left.=3.02 \times 10^{-4} \mathrm{M}\right)$, (c) pyrene in EPC vesicles $\left([\mathrm{PY}]=6.53 \times 10^{-5} \mathrm{M}\right)$, and $(\mathrm{d})$ E05P10 solution mixed with EPC vesicles $\left([\mathrm{PY}]=6.27 \times 10^{-5} \mathrm{M},[\mathrm{EAK}]=3.42 \times 10^{-6} \mathrm{M}.\right), \lambda_{\mathrm{ex}}=336 \mathrm{~nm}$.

pyrene from E05P10 solution into EPC vesicles with the final pyrene concentrations of $1.0 \times 10^{-4} \mathrm{M}$. Profile 2 obtained at higher pyrene concentration gives a sharp increase up to a maximum of $I_{m}$ with time after 0.1 hour, followed by a continuous decrease down to a plateau reached after about 3.5 hours. The fluorescence behavior in Figure 2 indicates that the transition undergone by pyrene from microcrystals of the E05P10 solution to individual molecules dissolved inside the lipid bilayer. Inside a crystal, pyrene molecules are very close to each other, and the probability of pyrene molecules to absorb a photon is small, while an equal number of pyrene molecules are dissolved inside the vesicle as a molecular state, and the probability of pyrene to absorb a photon becomes much bigger. As the concentration of pyrene inside the vesicle membrane increased, the pyrene absorption increases and so does the fluorescence of the pyrene monomer, which in turn results in the formation of more excimer inside the membrane [19]. As shown in profile 1 of Figure $4, I_{m}$ reaches a plateau when all the pyrene is molecularly dissolved inside the vesicles at a lower final pyrene concentration. If the amount of pyrene (aliquots of the EAKI-PY solution) added to the liposome solution is too large, the dissolution of pyrene molecules in the lipid vesicles leads to a rapidly increasing of pyrene absorption. After a critical point of the pyrene concentration in liposome, the pyrene absorption becomes too large and the inner filter effect takes place [20], which leads to the decreasing of the pyrene monomer fluorescence until the crystals are fully dissolved and a plateau is reached. This is why the final pyrene concentration of profile 2 in Figure 4 is 8 times larger than that of profile 1 .

Quantitative analysis of $I_{m} / I_{s}$-versus-time profiles such as those shown in Figure 4 was required to obtain detail information of the pyrene transfer velocity from peptide 


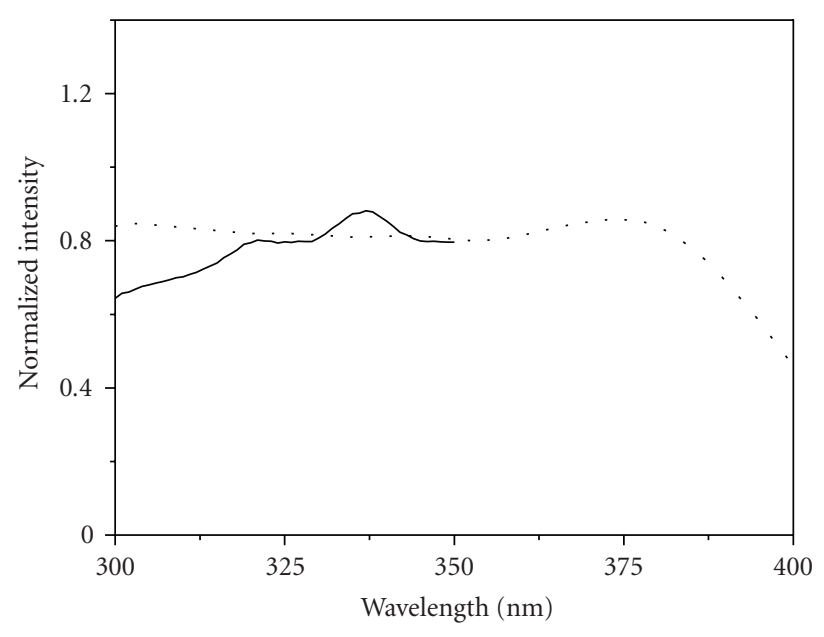

(a)

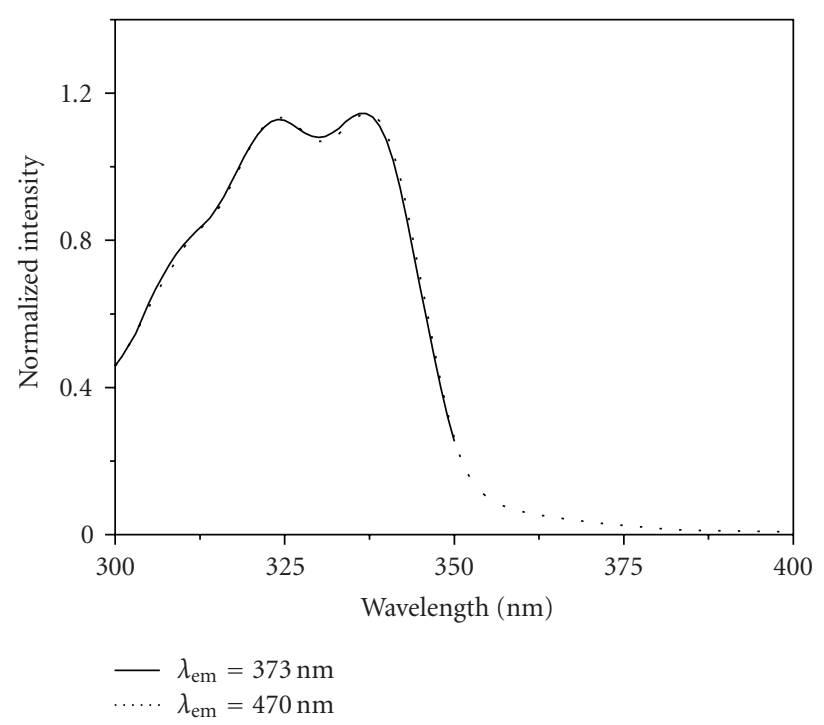

(c)

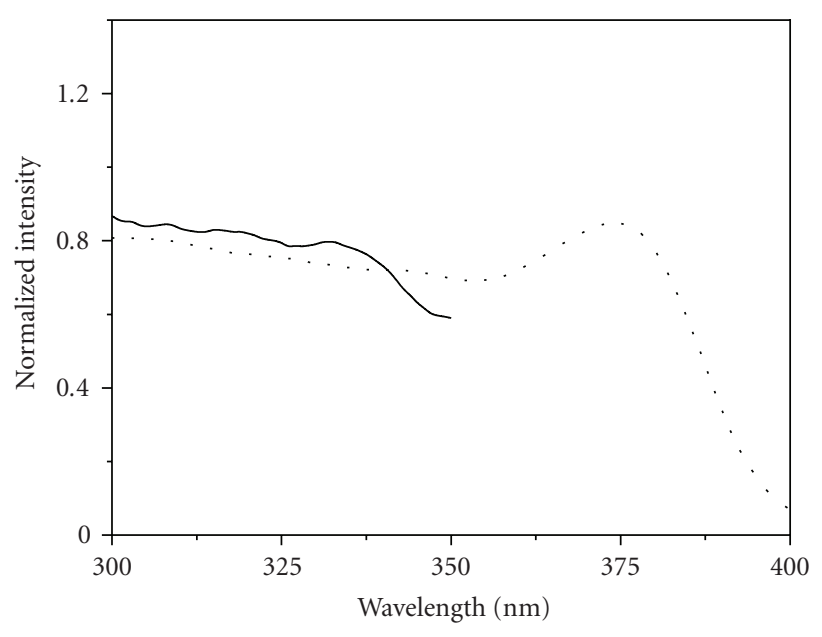

(b)

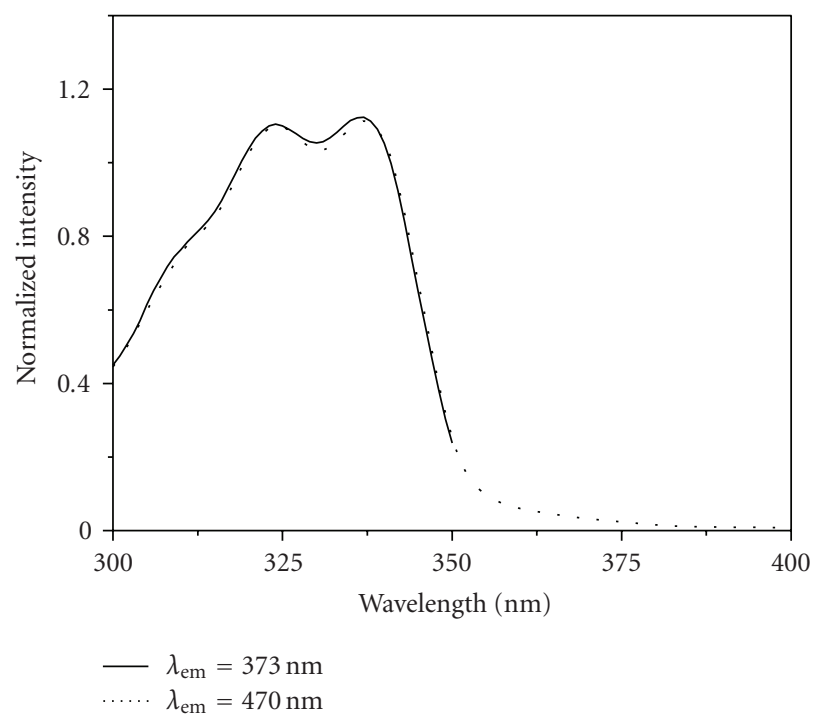

(d)

Figure 3: Normalized steady-state fluorescence excitation spectra of (a) solid pyrene crystals, (b) E05P10 solution ([PY] = 4.94 $\times 10^{-3} \mathrm{M}$, $[$ EAK16-I $\left.]=3.02 \times 10^{-4} \mathrm{M}\right)$, (c) pyrene in EPC vesicles $\left([\mathrm{PY}]=6.53 \times 10^{-5} \mathrm{M}\right)$, and $(\mathrm{d})$ E05P10 solution mixed with EPC vesicles $\left([\mathrm{PY}]=6.27 \times 10^{-5} \mathrm{M},[\mathrm{EAK} 16-\mathrm{I}]=3.42 \times 10^{-6} \mathrm{M}\right.$.), Emission wavelength $\left(\lambda_{\mathrm{em}}\right)$ was $373 \mathrm{~nm}(-)$ and $468 \mathrm{~nm}(\cdots)$.

encapsulation to vesicle membranes. Therefore, the calibration curve of $I_{m}$-versus-concentration of pyrene in EPC vesicles was needed (see Figure 5). A set of EPC-PY solutions was prepared with the pyrene concentrations ranging from $2 \times 10^{-6} \mathrm{M}$ to $1.2 \times 10^{-4} \mathrm{M}$. To fit the pyrene transfer experiments in different days, $I_{m}$ of these solutions was divided by $I_{s}$ to avoid xenon lamp fluctuations. The calibration curves also show the inner filter effect obviously. $I_{m} / I_{s}$ passes through a maximum, and its value varies little for pyrene concentration between $2.2 \times 10^{-5} \mathrm{M}$ and $5.3 \times 10^{-5} \mathrm{M}$. Consequently, there is a gap observed in time-dependent concentration profile for pyrene located inside the vesicles, where no data points are reported for pyrene concentrations ranging from $2.2 \times 10^{-5}$ to $5.3 \times 10^{-5} \mathrm{M}$. Therefore, two functions of $I_{m} / I_{s}$ fitted for different pyrene concentration range were get, and both of them were fit with sigmoidal. Up to $[\mathrm{PY}]=3.5 \times 10^{-5} \mathrm{M}$, the monomer intensity was fit with one sigmoidal, $I_{m} / I_{s}=$ $3.077-23.834 /\left(1+e^{([\mathrm{PY}]-6.460) / 8.678}\right)\left(R^{2}=0.9982\right)$. Another sigmoidal, $I_{m} / I_{s}=1.762+1.722 /\left(1+e^{([\mathrm{PY}]-63.341) / 27.522}\right)\left(R^{2}=\right.$ 0.9867 ) was used to fit with the monomer intensity for higher pyrene concentrations.

By transforming $I_{m} / I_{s}$-versus-time profiles with calibration curve of $I_{m} / I_{s}$-versus-concentration of pyrene in EPC, we got a release rate of pyrene from its peptide carrying systems into EPC vesicles (see Figure 6). The molecular ratios of peptide-to-pyrene used in E05P02 and E05P10 solutions are 0.229 and 0.061 , respectively. For similar final pyrene concentrations in the EPC vesicles, the release of pyrene from the EAK16-I coated pyrene crystals in E05P02 solution proceeds in a slower manner than that in E05P10 solution. 


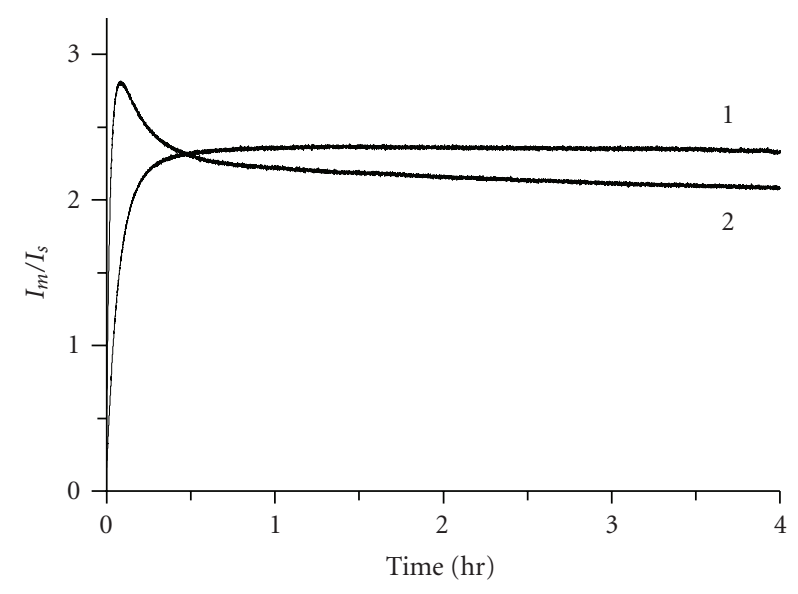

FIgURE 4: Fluorescence emission of the pyrene monomer at emission wavelength $373 \mathrm{~nm}$ when different amounts of E05P10 solution are added to EPC vesicle solutions. The fluorescence intensity $\left(I_{m}\right)$ is divided by that of the standard $\left(I_{s}\right)$. Data shown in profile $1\left([\mathrm{PY}]=1.3 \times 10^{-5} \mathrm{M},[\mathrm{EAK} 16-\mathrm{I}]=8.05 \times 10^{-7} \mathrm{M}\right)$ and profile $2\left([\mathrm{PY}]=1.1 \times 10^{-4} \mathrm{M}\right.$, [EAK16-I $\left.]=5.13 \times 10^{-6} \mathrm{M}\right)$ were both acquired over a 4 -hour time span with 0.2 -second intervals.

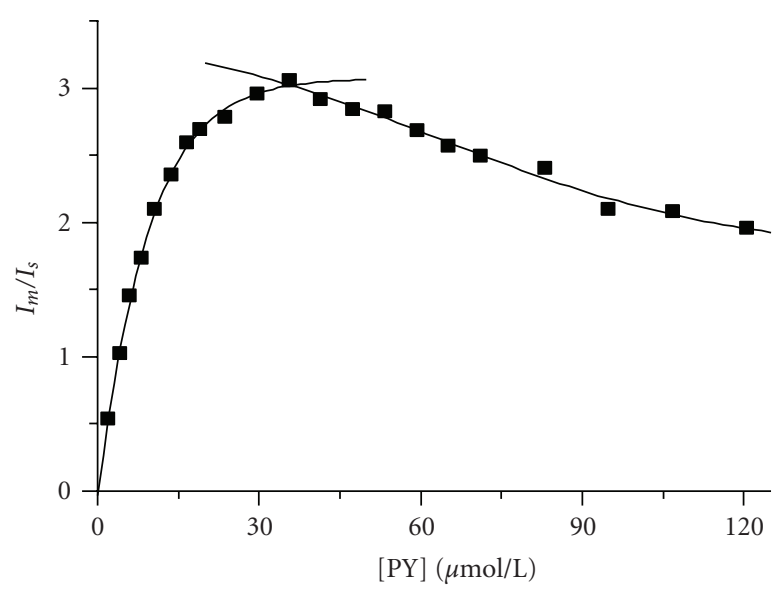

FIGURE 5: Calibration curve for different pyrene concentrations in EPC vesicles $\left([\mathrm{EPC}]=7.32 \times 10^{-4} \mathrm{M}\right)$. The concentration of pyrene ranged from $2 \mu \mathrm{M}$ to $120 \mu \mathrm{M}$. The fluorescence intensity $\left(I_{m}\right)$ is divided by that of the standard $\left(I_{s}\right)$. Up to $[\mathrm{PY}]=35 \mu \mathrm{M}$, the monomer intensity was fit with a sigmoidal $\left(R^{2}=0.9982\right), I_{m} / I_{s}=$ $3.077-23.834 /\left(1+e^{([\mathrm{PY}]-6.460) / 8.678}\right)$. Another sigmoidal $\left(R^{2}=0.9867\right.$ $I_{m} / I_{s}=1.762+1.722 /\left(1+e^{([\mathrm{PY}]-63.341) / 27.522}\right)$, was used to fit with the monomer intensity for higher Pyrene concentrations.

To quantify the release rate, the pyrene release constant was acquired. The trend shown in Figure 6 can be fitted with

$$
\left[\mathrm{PY}_{V}\right](t)=\left[\mathrm{PY}_{V}\right]_{\mathrm{eq}}-\left(\left[\mathrm{PY}_{V}\right]_{\mathrm{eq}}-\left[\mathrm{PY}_{V}\right]_{0}\right) \exp (-k \times t)
$$

In $(1),\left[\mathrm{PY}_{V}\right](t),\left[\mathrm{PY}_{V}\right]_{\mathrm{eq}}$, and $\left[\mathrm{PY}_{V}\right]_{0}$ represent the pyrene concentration inside the vesicles at time $t$, at equilibrium (infinite time), and at time $t=0$ seconds, respectively. According to (1), the release rate constants $(k)$ of different

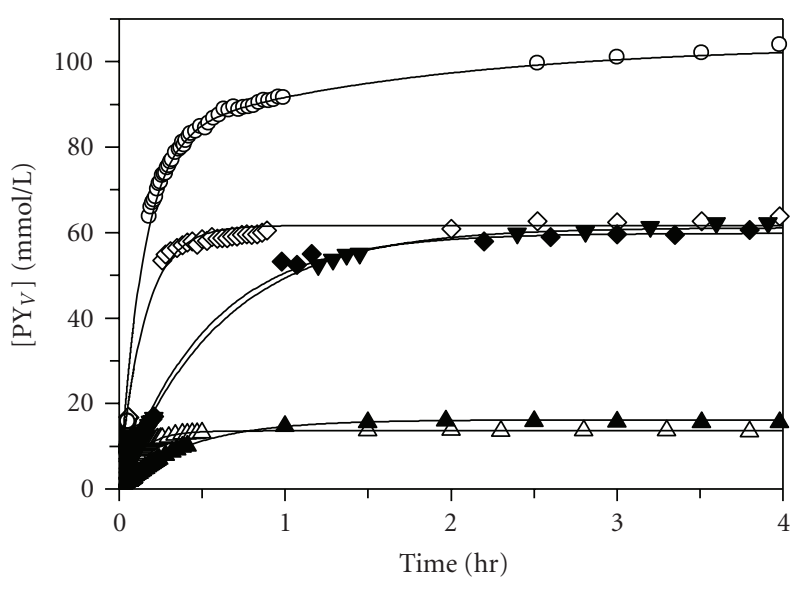

FIGURE 6: Profiles for the release of molecular pyrene from EAK16I coated pyrene microcrystals into a solution of EPC vesicles according to the fluorescence results. Hollow and solid symbols are for pyrene transfer experiments carried out with E05P10 and E05P02, respectively. The final pyrene concentrations are as follows: $(\circ)[\mathrm{PY}]=1.04 \times 10^{-4} \mathrm{M} ;(\diamond)[\mathrm{PY}]=6.37 \times 10^{-5} \mathrm{M} ;(\square)[\mathrm{PY}]=$ $1.33 \times 10^{-5} \mathrm{M} ;(\diamond)[\mathrm{PY}]=6.14 \times 10^{-5} \mathrm{M} ;(\boldsymbol{\nabla})[\mathrm{PY}]=6.7 \times 10^{-5} \mathrm{M}$ (from settled E05P02 solution); $(\mathbf{\Delta})[\mathrm{PY}]=1.56 \times 10^{-5} \mathrm{M}$.

peptide-pyrene-EPC systems were shown in Table 1. This suggests that the release rate can be controlled by changing peptide-cargo ratio.

The difference of release rates of pyrene from pyrene crystals coated with EAK16-I can be related to a thicker encapsulation of the pyrene crystals when a higher molecular ratio of peptide-to-pyrene was used. As shown in Figure 7, the sizes of the peptide-pyrene complexes in two EAKI-PY colloidal suspensions are both several micrometers, but the surfaces of the pyrene crystals in the E05P10 (see Figure 7(a)) and E05P02 (see Figure 7(b)) solutions exhibit different appearances depending on the peptide-to-pyrene ratio. Pyrene crystals imaged from the E05P10 solution display a thick encapsulation, while most of the pyrene crystals imaged from the E05P02 solution appear to be wrapped in an even thicker encapsulation. XPS spectra can offer elements ratios of surface layers. The $\mathrm{C}$ elements of the peptide-pyrene complexes surface layers contributed by peptide and pyrene, while the $\mathrm{N}$ elements of those contributed by peptide only. Thicker encapsulation meant more peptide in the surface layers, which led to lower $\mathrm{C}$-to- $\mathrm{N}$ ratio. The molecular ratios of C-to-N of the E05P02 and E05P10 complexes surface layers were 5.965 and 6.494, respectively. This indicated that the encapsulation of the complexes from E05P02 was thicker that from E05P10. The micrographs shown in Figure 7 and the molecular ratios of $\mathrm{C}$-to- $\mathrm{N}$ suggested that higher peptideto-pyrene molecular ratios lead to the formation of a thicker peptide encapsulation which inhibits the release of pyrene.

The EAKI-PY colloidal suspensions were found to settle over time without continuous stirring. To investigate whether the settling of the colloids has an effect on the pyrene release rate, a release experiment was conducted with the resuspended E05P02 solution which was settled over a period of $5 \mathrm{~d}$ after the equilibrium was reached and 
TABLE 1: Transfer rates of molecular pyrene from EAK16-I coated pyrene microcrystals into a solution of EPC vesicles.

\begin{tabular}{lcl}
\hline Molecular ratios of EAK16-I-to-pyrene ${ }^{(\mathrm{a})}$ & {$[\mathrm{PY}]^{(\mathrm{b})}\left(\mu \mathrm{mol} \cdot L^{-1}\right)$} & $\begin{array}{l}\text { Rate constants of transfer } \\
(k)\left(\mathrm{h}^{-1}\right)\end{array}$ \\
\hline 0.061 & 13.3 & $7.92 \pm 0.01$ \\
0.229 & 15.6 & $2.30 \pm 0.01$ \\
0.061 & 63.7 & $6.52 \pm 0.06$ \\
0.229 & 61.4 & $1.85 \pm 0.01$ \\
0.229 & $62.6^{(\mathrm{d})}$ & $1.63 \pm 0.01$ \\
0.061 & $104.2^{(\mathrm{c})}$ & fast: $7.10 \pm 0.09(78.11 \%) ;$ \\
\hline
\end{tabular}

(a) molecular ratios of EAK16-I-to-pyrene for E05P10 (0.061) and E05P02 (0.229) solution.

(b) pyrene concentrations in the solutions of EPC vesicles at the end points of each transfer experiment.

(c) two exponentials were needed to fit the release profile according to $[\mathrm{PY}](t)=[\mathrm{PY}]_{(t=\infty)} \times\left[1-p_{\text {fast }} \times \exp \left(-k_{\text {fast }} t\right)-p_{\text {slow }} \times\right.$ $\left.\exp \left(-k_{\text {slow }} t\right)\right]$ where $p_{\text {fast }}$ and $p_{\text {slow }}$ represent the proportion of fast release and slow release in overall release, respectively, and $p_{\text {fast }}+p_{\text {slow }}=100 \%$.

(d) from settled E05P02 solution.

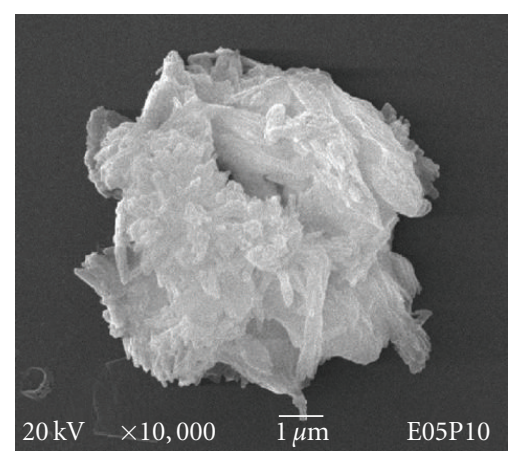

(a)

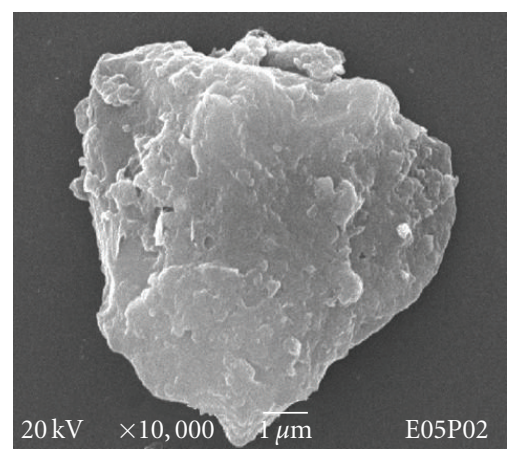

(b)

FIGURE 7: Scanning electron micrographs of the pyrene crystals of (a) the E05P10 solution and (b) the E05P02 solution.

shaken vigorously before release experiment. The results are shown in Figure 6 and Table 1. Within experimental error, no difference was observed in the release profiles, whether the release experiment was performed with the settled or continuously stirred E05P02 solution.

\section{CONCLUSION}

This study has demonstrated that the mixing of a model hydrophobic compound with self-assembling EAK16-I peptide in aqueous solution involving mechanical stirring resulted in the formation of colloidal suspensions. EAK16I acted as a colloidal stabilizer which interacted with the surface of crystalline form rather than molecular state pyrene. When the EAKI-PY solution was mixed with EPC vesicles, pyrene was transferred from the pyrene crystals into the vesicle membrane until all pyrene crystals had disappeared. Steady-state fluorescence showed that pyrene was molecularly dissolved inside the vesicle membrane. The release behavior of pyrene from its EAK16-I coating was studied by following the fluorescence of pyrene upon exposure to EPC vesicles as a function of time. The transfer rate constants were determined and found to be much slower when pyrene was released from the E05P02 solution than from the E05P10 solution. Scanning electron micrographs and $\mathrm{C}$-to- $\mathrm{N}$ ratios of the complexes surface layers suggested that this effect was due to thicker peptide coating which inhibited the release of pyrene displayed in E05P02 solution. In conclusion, our results suggested that the amphiphilic selfassembling peptide EAK16-I had the potential to become a carrier for low molecular weight, hydrophobic compounds whose release into target cells could be controlled by tuning the peptide-to-cargo molecular ratio.

\section{ACKNOWLEDGMENTS}

We thank Professor Pu Chen of the University of Waterloo for his helpful suggestions and stimulating discussions. The support of Dr. Zhaoyang Ye for preparing this manuscript was especially and gratefully acknowledged. This work was supported by the Chinese National "985 Project" of Education Ministry based in Sichuan University, and by the Analytical and Testing Center of Sichuan University for providing technique support, respectively, Chengdu, China. 


\section{REFERENCES}

[1] T. Gershanik and S. Benita, "Self-dispersing lipid formulations for improving oral absorption of lipophilic drugs," European Journal of Pharmaceutics and Biopharmaceutics, vol. 50, no. 1, pp. 179-188, 2000.

[2] X. Zhao and S. Zhang, "Molecular designer self-assembling peptides," Chemical Society Reviews, vol. 35, no. 11, pp. 11051110, 2006.

[3] S. Zhang, T. C. Holmes, C. Lockshin, and A. Rich, "Spontaneous assembly of a self-complementary oligopeptide to form a stable macroscopic membrane," Proceedings of the National Academy of Sciences of the United States of America, vol. 90, no. 8, pp. 3334-3338, 1993.

[4] S. Zhang, T. C. Holmes, C. M. DiPersio, R. O. Hynes, X. $\mathrm{Su}$, and A. Rich, "Self-complementary oligopeptide matrices support mammalian cell attachment," Biomaterials, vol. 16, no. 18, pp. 1385-1393, 1995.

[5] J. Kisiday, M. Jin, B. Kurz, et al., "Self-assembling peptide hydrogel fosters chondrocyte extracellular matrix production and cell division: implications for cartilage tissue repair," Proceedings of the National Academy of Sciences of the United States of America, vol. 99, no. 15, pp. 9996-10001, 2002.

[6] S. R. Whaley, D. S. English, E. L. Hu, P. F. Barbara, and A. M. Belcher, "Selection of peptides with semiconductor binding specificity for directed nanocrystal assembly," Nature, vol. 405, no. 6787, pp. 665-668, 2000.

[7] Y. Nagai, L. D. Unsworth, S. Koutsopoulos, and S. Zhang, "Slow release of molecules in self-assembling peptide nanofiber scaffold," Journal of Controlled Release, vol. 115, no. 1, pp. 18-25, 2006

[8] C. Keyes-Baig, J. Duhamel, S.-Y. Fung, J. Bezaire, and P. Chen, "Self-assembling peptide as a potential carrier of hydrophobic compounds," Journal of the American Chemical Society, vol. 126, no. 24, pp. 7522-7532, 2004.

[9] S. Y. Fung, H. Yang, and P. Chen, "Formation of colloidal suspension of hydrophobic compounds with an amphiphilic self-assembling peptide," Colloids and Surfaces B, vol. 55, no. 2, pp. 200-211, 2007.

[10] S. Jun, Y. Hong, H. Imamura, B.-Y. Ha, J. Bechhoefer, and P. Chen, "Self-assembly of the ionic peptide EAK16: the effect of charge distributions on self-assembly," Biophysical Journal, vol. 87, no. 2, pp. 1249-1259, 2004.

[11] Y. Hong, L. S. Lau, R. L. Legge, and P. Chen, "Critical selfassembly concentration of an ionic-complementary peptide EAK16-I," The Journal of Adhesion, vol. 80, no. 10-11, pp. 913$931,2004$.

[12] Y. Xu and F. C. Szoka Jr., "Mechanism of DNA release from cationic liposome/DNA complexes used in cell transfection," Biochemistry, vol. 35, no. 18, pp. 5616-5623, 1996.

[13] M. Wilhelm, C.-L. Zhao, Y. Wang, et al., "Poly(styreneethylene oxide) block copolymer micelle formation in water: a fluorescence probe study," Macromolecules, vol. 24, no. 5, pp. 1033-1040, 1991.

[14] G. P. L'Heureux and M. Fragata, "Micropolarities of lipid bilayers and micelles. 5. Localization of pyrene in small unilamellar phosphatidylcholine vesicles," Biophysical Chemistry, vol. 30, no. 3, pp. 293-301, 1988.

[15] S. Pandey, R. A. Redden, A. E. Hendricks, K. A. Fletcher, and C. P. Palmer, "Characterization of the solvation environment provided by dilute aqueous solutions of novel siloxane polysoaps using the fluorescence probe pyrene," Journal of Colloid and Interface Science, vol. 262, no. 2, pp. 579-587, 2003.
[16] C. Gao, H. Qian, S. Wang, D. Yan, W. Chen, and G. Yu, "Selfassociation of hyperbranched poly(sulfone-amine) in water: studies with pyrene-fluorescence probe and fluorescence label," Polymer, vol. 44, no. 5, pp. 1547-1552, 2003.

[17] M. R. Vigil, J. Bravo, T. D. Z. Atvars, and J. Baselga, "Photochemical sensing of semicrystalline morphology in polymers: pyrene in polyethylene," Macromolecules, vol. 30, no. 17 , pp. 4871-4876, 1997.

[18] J.-H. Kim, M. M. Domach, and R. D. Tilton, "Pyrene micropartitioning and solubilization by sodium dodecyl sulfate complexes with poly(ethylene glycol)," Journal of Physical Chemistry B, vol. 103, no. 48, pp. 10582-10590, 1999.

[19] D. Daems, M. Van den Zegel, N. Boens, and F. C. De Schryver, "Fluorescence decay of pyrene in small and large unilamellar L, $\alpha$-dipalmitoylphosphatidylcholine vesicles above and below the phase transition temperature," European Biophysics Journal, vol. 12, no. 2, pp. 97-105, 1985.

[20] S. A. French, P. R. Territo, and R. S. Balaban, "Correction for inner filter effects in turbid samples: fluorescence assays of mitochondrial NADH," American Journal of Physiology, vol. 275, no. 3, pp. C900-C909, 1998. 

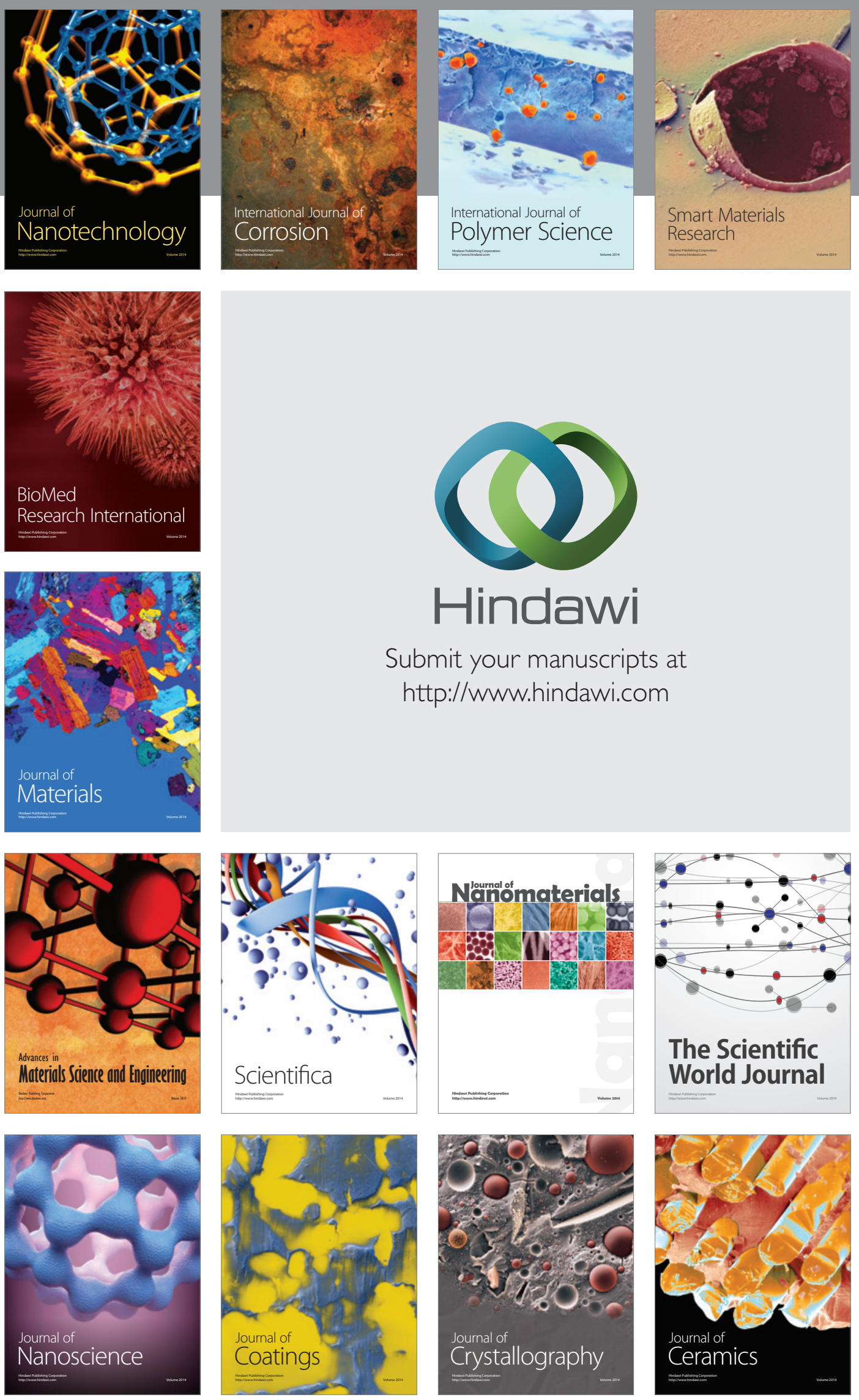

The Scientific World Journal

Submit your manuscripts at

http://www.hindawi.com

\section{World Journal}

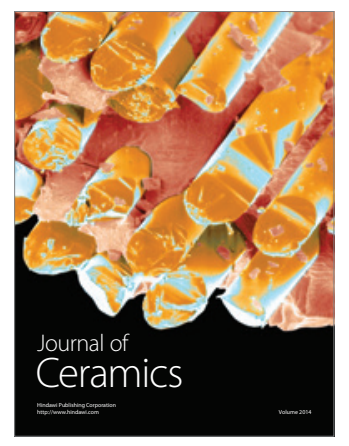

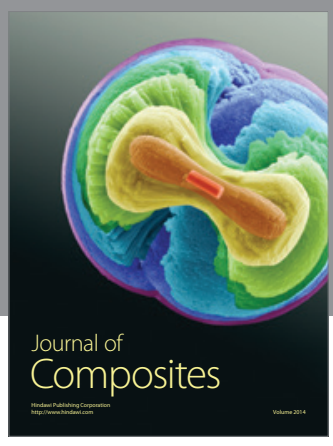
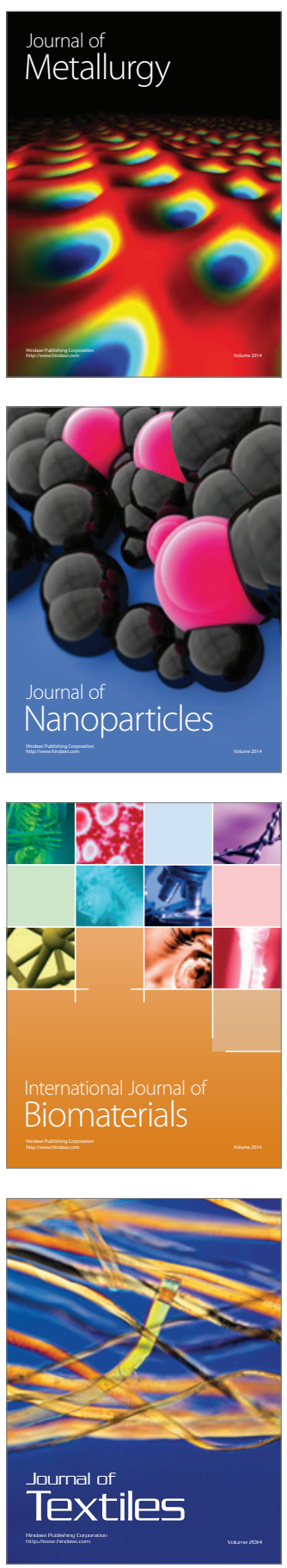